\title{
Potential predictors of survival after surgery for colorectal cancer patients with synchronous unresectable liver metastases
}

\author{
TSUKASA HOTTA, KATSUNARI TAKIFUJI, KAZUHISA UCHIYAMA, SHOZO YOKOYAMA, KENJI MATSUDA, \\ TAKASHI HIGASHIGUCHI, TOSHIJI TOMINAGA, YOSHIMASA OKU, TORU NASU and HIROKI YAMAUE
}

Second Department of Surgery, Wakayama Medical University, School of Medicine, Wakayama, Japan

Received June 20, 2006; Accepted September 15, 2006

\begin{abstract}
Liver resection has been recognized as the best treatment for patients with colorectal liver metastases, but as a curative resection for multiple and bilobar colorectal liver metastases (MBCLM) it is definitely less effective. We clarify predictors of survival for unresectable MBCLM. Potential predictors of overall survival, and the correlation between tumor marker and survival were evaluated for patients with synchronous unresectable MBCLM, including 6 rectal and 17 colon cancers. In univariate analysis, survival in patients with the following parameters were longer than those without them: number of liver metastases $(\leq 10)$, without lung metastasis and peritoneal invasion, and with a $<1.0$ ratio of postoperative CEA/preoperative CEA. In multivariate analysis, the numbers of liver metastases $(>10)$ and a $>1.0$ ratio of postoperative $\mathrm{CEA}$ /preoperative CEA were factors of poor prognosis, and patients with two such factors had an even worse prognosis. There was a tendency for correlation between the ratio of postoperative CEA/pre-operative CEA and survival ( $\mathrm{R}=-0.492, \mathrm{P}=0.053 ; \mathrm{y}=17.388-3.733 \mathrm{x})$. Thus, we clarified some of the predictors of survival for MBCLM, and the usefulness of serum CEA.
\end{abstract}

\section{Introduction}

Colorectal cancer is one of the most common causes of malignancy-related death in the United States, Japan and European countries $(1,2)$. In patients with untreated colorectal liver metastases, the 1-, 2-, 3- and 4-year survival rates were reported to be $31,7.9,2.6$ and $0.9 \%$, respectively (3). Liver resection has been recognized as the best treatment for long-term survival in patients with colorectal liver metastases (4). The 5-year survival rate after a liver resection in patients with colorectal liver metastases has been reported

Correspondence to: Dr Hiroki Yamaue, Second Department of Surgery, Wakayama Medical University, School of Medicine, 811-1 Kimiidera, Wakayama 641-8510, Japan

E-mail: yamaue-h@wakayama-med.ac.jp

Key words: colorectal cancer, liver metastasis, prognostic factor to be $20-50 \%(5,6)$. On the other hand, liver resection can be performed only in approximately $10-20 \%$ of patients with colorectal liver metastases, because a liver resection is contraindicated due to unresectable multiple and bilobar metastasis (7). One report demonstrated that no patient with three or more metastatic lesions has survived disease-free for more than 48 months (8).

In this study, we tried to demonstrate the potential predictors of survival after surgery for colorectal cancer patients with synchronous unresectable MBCLM.

\section{Patients and methods}

Patients. Twenty-three stage IV colorectal cancer patients with synchronous unresectable multiple and bilobar colorectal liver metastases (MBCLM) who underwent surgery at Wakayama Medical University Hospital between December 1999 and August 2004 were enrolled in this study. Data on these patients were collected and followed up from December 1999 to December 2004. Six patients had rectal cancer and 17 had colon cancer including; 2 cecum, 4 ascending, 1 transverse and 10 sigmoid colons. TNM clinical stage IV is described according to the UICC classification of malignant tumors (9). We defined that unresectable 5 or more lesion metastases to both lobes of the liver was unresectable MBCLM according to the definition of $\mathrm{H} 3$ liver metastasis by Japanese classification of colorectal carcinoma (10).

A resection of the primary tumor in the rectum and colon was performed with a lymphadenectomy from along the rectal or large intestinal wall to around the main feeding artery.

Intra-operative evaluation of liver metastases status was performed by palpation, intra-operative ultrasonography (US), and frozen-section microscopic examination. There were no patients with a liver resection, treatment of radiation therapy or neoadjuvant chemotherapy in this study.

The course of postoperative $l$-LV/5FU chemotherapy consisted of a weekly administration of $l$-LV and $5 \mathrm{FU}$ for 6 weeks, in 2-week intervals. During postoperative chemotherapy, $250 \mathrm{mg} / \mathrm{m}^{2}$ of $l$-LV (Wyeth Co., Tokyo, Japan) was drip intravenous infused for $2 \mathrm{~h}$, and $600 \mathrm{mg} / \mathrm{m}^{2}$ of $5 \mathrm{FU}$ (Kyowa Hakko, Tokyo, Japan) was administered $1 \mathrm{~h}$ after the start of $l$-LV administration as follows; $250 \mathrm{mg}$ of $5 \mathrm{FU}$ was administered through the catheter of hepatic intra-arterial infusion chemotherapy, and the remnant dose of 5FU (600 mg/ $\mathrm{m}^{2}-250 \mathrm{mg}$ ) was given intravenously $1 \mathrm{~h}$ after the start of $l$-LV 
Table I. Univariate analysis of potential predictors of survival after surgery.

\begin{tabular}{|c|c|c|c|c|}
\hline \multirow[b]{2}{*}{ Variable } & \multicolumn{2}{|c|}{ Overall survival (\%) } & \multirow[b]{2}{*}{ MST (months) } & \multirow[b]{2}{*}{ P-value } \\
\hline & 1-year & 2-year & & \\
\hline Age (years) & & & & 0.479 \\
\hline$<65(\mathrm{n}=10)$ & 39.4 & 0 & 11.0 & \\
\hline$\geq 65(\mathrm{n}=13)$ & 60.6 & 25.2 & 19.0 & \\
\hline Gender & & & & 0.195 \\
\hline Male $(n=16)$ & 59.6 & 31.8 & 18.0 & \\
\hline Female $(n=7)$ & 35.7 & 0 & 11.0 & \\
\hline Primary lesion & & & & 0.098 \\
\hline Colon $(n=17)$ & 46.7 & 0 & 11.0 & \\
\hline Rectum (n=6) & 66.7 & 44.4 & 22.0 & \\
\hline Histopathological type & & & & 0.407 \\
\hline Well differentiated $(n=13)$ & 65.8 & 16.5 & 18.0 & \\
\hline Other type $(n=10)$ & 36.0 & 24.0 & 9.0 & \\
\hline Depth of invasion & & & & 0.997 \\
\hline ss, a1 (n=15) & 54.5 & 21.8 & 14.0 & \\
\hline se, a2, si (n=8) & 50.0 & 0 & 11.0 & \\
\hline Lymph node metastasis & & & & 0.380 \\
\hline N0, $1(n=17)$ & 57.8 & 26.4 & 14.0 & \\
\hline $\mathrm{N} 2 \leq(\mathrm{n}=6)$ & 27.8 & 0 & 11.0 & \\
\hline Lymphatic invasion & & & & 0.187 \\
\hline $1,2(\mathrm{n}=18)$ & 56.3 & 30.0 & 18.0 & \\
\hline $3(n=5)$ & 40.0 & 0 & 11.0 & \\
\hline Venous invasion & & & & 0.926 \\
\hline $1,2(\mathrm{n}=18)$ & 50.6 & 27.0 & 14.0 & \\
\hline $3(n=5)$ & 60.0 & 0 & 18.0 & \\
\hline No. of liver metastasis & & & & 0.003 \\
\hline$\leq 10(\mathrm{n}=14)$ & 76.2 & 31.7 & 19.0 & \\
\hline$>10(\mathrm{n}=9)$ & 22.2 & 0 & 9.0 & \\
\hline Maximum size of liver metastasis & & & & 0.081 \\
\hline$<100 \mathrm{~mm}(\mathrm{n}=18)$ & 62.5 & 22.8 & 18.0 & \\
\hline$\geq 100 \mathrm{~mm}(\mathrm{n}=5)$ & $\mathrm{NC}$ & & 5.0 & \\
\hline Lung metastasis & & & & 0.002 \\
\hline$-(n=19)$ & 64.8 & 23.6 & 18.0 & \\
\hline$+(n=4)$ & 0 & & 3.0 & \\
\hline Peritoneal invasion & & & & 0.043 \\
\hline$-(n=20)$ & 62.2 & 22.7 & 18.0 & \\
\hline$+(n=3)$ & 0 & & 9.0 & \\
\hline Serum CEA level & & & & 0.019 \\
\hline$<1.0(\mathrm{n}=7)$ & 71.4 & 47.6 & 22.0 & \\
\hline$>1.0(\mathrm{n}=16)$ & 44.2 & 0 & 11.0 & \\
\hline Serum CA19-9 level & & & & 0.077 \\
\hline$<1.0(\mathrm{n}=7)$ & 68.6 & 45.7 & 22.0 & \\
\hline$>1.0(\mathrm{n}=16)$ & 45.5 & 0 & 11.0 & \\
\hline
\end{tabular}

MST, median survival time; NC, not able to calculate; Well differentiated, well differentiated adenocarcinoma; ss, tumor invasion of subserosa; se, tumor penetration of serosa; si, tumor invasion of adjacent structures; a1, tumor invasion through muscularis propria into nonperitonealized part; a2, tumor invasion of non-peritonealized, pericolic or perirectal tissues; N0, no regional lymph node metastasis; N1, metastasis in 1-3 regional lymph nodes; N2, metastasis in 4 or more regional lymph nodes. Lymphatic invasion 1, 2 and 3 means minimal, moderate and severe lymphatic invasion, respectively. Venous invasion 1, 2 and 3 means minimal, moderate and severe venous invasion, respectively. $<1.0$, the ratio of postoperative tumor marker level/preoperative tumor marker level was $<1.0$. 
administration. This treatment was designated as a 'modified RPMI regimen for liver metastasis' because of the administration of $l$-LV alone, compared with use of racemic leucovorin $(d, l-\mathrm{LV})$ in the original RPMI regimen $(11,12)$. When $l$-LV/ 5FU chemotherapy was not effective and an additional agent was tolerable due to the lack of severe side-effects, additional CPT-11 administration was performed, that is, $100 \mathrm{mg}$ of irinotecan (CPT-11) (Daiichi Pharmaceutical Co., Ltd., Tokyo, Japan) was administered intravenously every 2 weeks during $l$-LV/5FU chemotherapy ( $l$-LV/5FU to CPT- 11$)$.

Age, gender, primary lesion, histopathological type, depth of invasion, lymph node metastasis, lymphatic invasion, venous invasion, number of liver metastasis, maximum size of liver metastasis, lung metastasis, peritoneal invasion, serum carcinoembryonic antigen (CEA) level and serum carbohydrate antigen (CA) 19-9 level were chosen as the candidates for prognostic factors. The ages of the patients ranged from 37 to 79 (median: 65 years), therefore, age factor was divided to $<65$ and $\geq 65$. The depths of invasion were defined as follows: ss, tumor invasion of subserosa; se, tumor penetration of serosa; si, tumor invasion of adjacent structures; a1, tumor invasion through muscularis propria into non-peritonealized part; a2, tumor invasion of non-peritonealized, pericolic or perirectal tissues. Lymph node metastasis was defined as: N0, no regional lymph node metastasis; N1, metastasis in 1-3 regional lymph nodes; N2, metastasis in 4 or more regional lymph nodes. Lymphatic invasion 1, 2 and 3 means minimal, moderate and severe lymphatic invasion, respectively. Venous invasion 1,2 and 3 means minimal, moderate and severe venous invasion, respectively. Lymphatic and venous invasion were evaluated according to Japanese classification of colorectal carcinoma (10).

Evaluation of liver metastasis status was performed by preoperative abdominal spiral computed tomography (CT) with bolus intravenous infusion and pre- and intra-operative US. Factors of the number of liver metastasis and the maximum size of liver metastasis were divided into two groups; over 10 or under 10 metastasis and larger or smaller than $100 \mathrm{~mm}$, respectively, because under 10 metastasis and smaller than $100 \mathrm{~mm}$ were considered to be accurately countable or measurable by CT and US.

Serum tumor marker levels including CEA and CA19-9 were evaluated by dividing into two groups as follows; the ratio of postoperative tumor marker level (data on 30th day after surgery)/preoperative tumor marker level of $>1.0$ or $<1.0$.

Patient follow-up. The mean and median follow-up periods of patients in this study were 30 and 28 months, respectively.

Statistical analysis. Statistical analysis was performed with Stat View-J ver. 5.0 using a Windows XP operating system. The overall survival rate for prognostic factors was estimated by the Kaplan-Meier method, and univariate analysis of significance for each factor was evaluated by a log-rank test. Multivariate analysis of the overall survival time was performed with Cox's proportional hazards model.

A P-value of $<0.05$ was considered statistically significant, and $<0.1$ and $\geq 0.05$ was considered a tendency to be statistically significant.
Table II. Estimation of overall survival adjusted on risk factors.

\begin{tabular}{|c|c|c|c|c|}
\hline \multirow[b]{2}{*}{$\begin{array}{l}\text { No. of positive } \\
\text { factor }\end{array}$} & \multicolumn{2}{|c|}{ Overall survival (\%) } & \multirow[b]{2}{*}{$\begin{array}{c}\text { MST } \\
\text { (months) }\end{array}$} & \multirow[b]{2}{*}{ P-value } \\
\hline & 1-year & 2-year & & \\
\hline & & & & 0.002 \\
\hline 0 Factor $(n=5)$ & 100 & 66.7 & 27.0 & \\
\hline 1 Factor $(n=11)$ & 40.9 & 0 & 11.0 & \\
\hline 2 Factors $(n=7)$ & 28.6 & 0 & 5.0 & \\
\hline
\end{tabular}

MST, median survival time; Factor, number of liver metastasis ( $>10)$ and serum CEA level (the ratio of postoperative CEA level/preoperative CEA level was $>1.0$ ).

\section{Results}

Analysis of potential predictors of survival after surgery. Survival in patients with $\leq 10$ liver metastases was longer than that in patients with $>10$ liver metastases $(\mathrm{P}=0.003)$. Survival in patients without lung metastasis and peritoneal invasion was longer than those in patients with them $(\mathrm{P}=0.002$ and 0.043 , respectively). Survival in patients, whose ratio of postoperative serum CEA level/preoperative serum CEA level was $<1.0$, was longer than patients whose ratio was $>1.0$ $(\mathrm{P}=0.019)$. On the other hand, there tended to be differences in primary lesion, maximum size of liver metastasis, and serum CA19-9 level ( $\mathrm{P}=0.098,0.081$ and 0.077 , respectively) (Table I).

Relative risk of overall survival was analyzed using Cox's proportional hazards model. There were 2 favorable factors that influenced overall survival after surgery. Survival in patients with $\leq 10$ liver metastases was longer than that in patients with $>10$ liver metastases $(\mathrm{P}=0.015$; $\mathrm{RR}, 25 ; 95 \% \mathrm{CI}$, 2-337). Next, survival in patients whose ratio of postoperative CEA level/preoperative serum CEA level was $<1.0$ was longer than that in patients whose ratio was $>1.0(\mathrm{P}=0.044$; RR, 4733; 95\% CI, 1-17584291). Fig. 1 shows the overall survival curves by the Kaplan-Meier method and the log-rank test value of these two factors.

Estimation of overall survival adjusted on risk factors. In order to estimate survival, two independent prognostic factors were used to adjust overall survival. Two independent factors of poor prognosis were number of liver metastasis $(>10)$ and serum CEA level (the ratio of postoperative CEA level/preoperative CEA level was $>1.0$ ).

There was a significant difference among survival of patients with no factors of poor prognosis (0 Factor), one poor factor (1 Factor), and two poor factors (2 Factor) $(\mathrm{P}=0.002)$ (Table II) (Fig. 2).

Correlation between the tumor marker and survival. Correlation between the tumor marker (postoperative serum CEA level/preoperative serum CEA level ratio, postoperative serum CA19-9 level/preoperative serum CA19-9 level ratio) and 

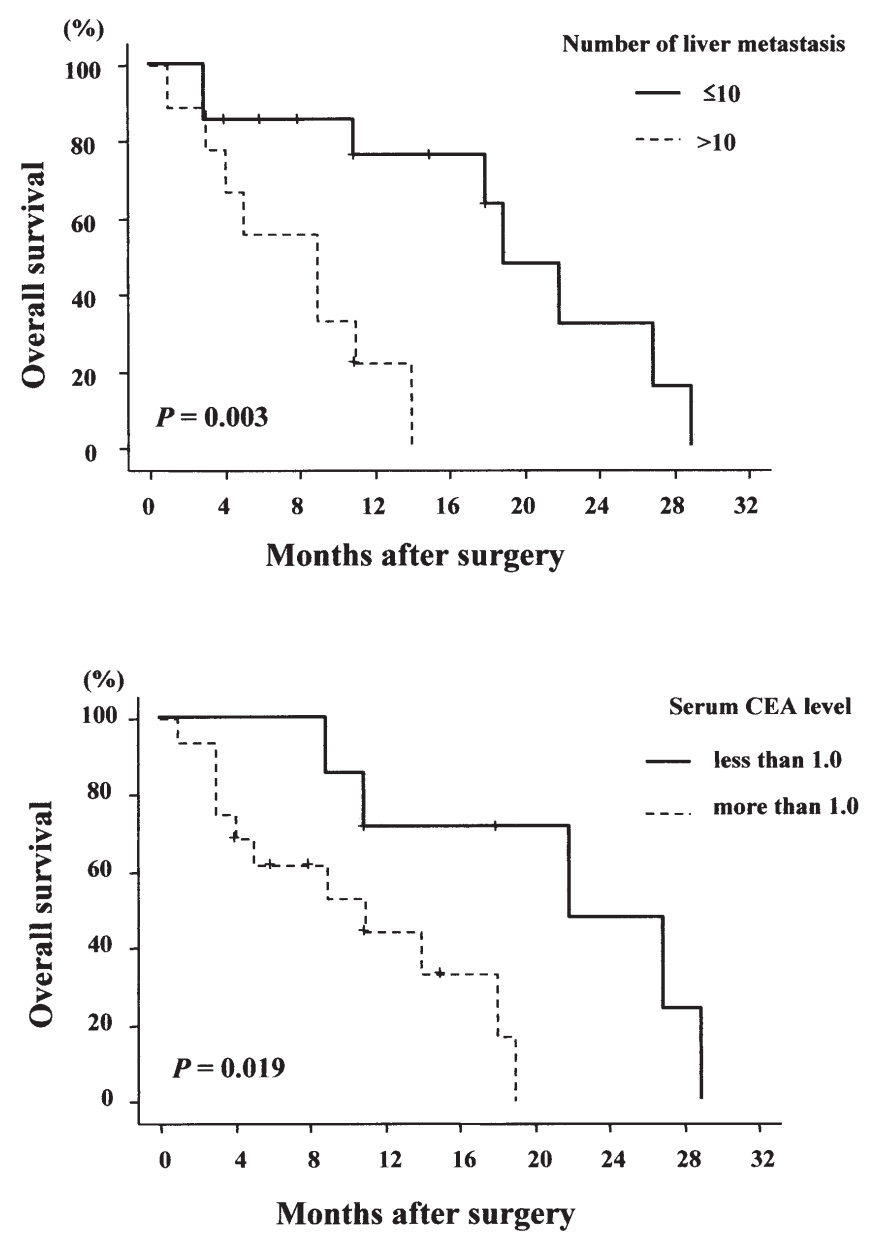

Figure 1. Overall survival curves by the Kaplan-Meier method in patients with a predictive prognostic factor. Survival in patients with $\leq 10$ liver metastases was longer than that in patients with $>10$ liver metastases $(\mathrm{P}=0.003)$. Survival in patients whose ratio of postoperative serum CEA level/preoperative CEA level was $<1.0$ was longer than that in patients whose ratio was $>1.0(\mathrm{P}=0.019)$.

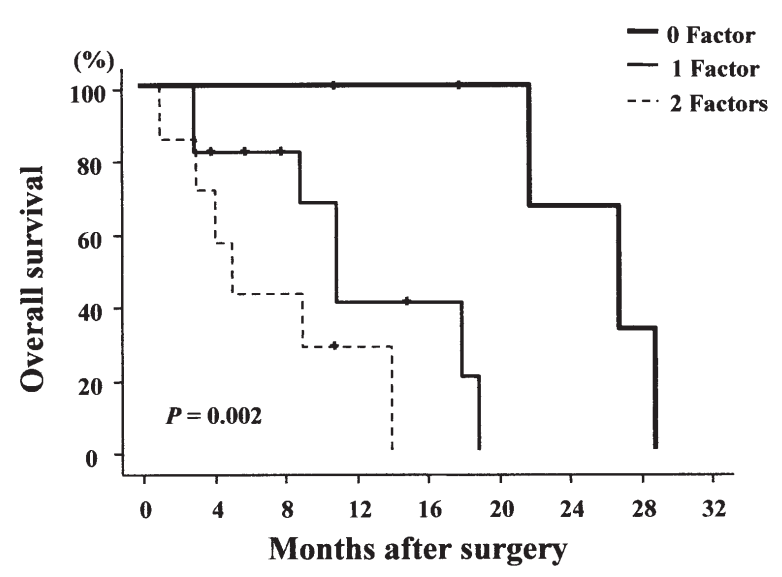

Figure 2. Overall survival curves by the Kaplan-Meier method in terms of the difference in the number of poor prognostic factors. There was a significant difference among survival of patients with no prognostic factor for poor prognosis (0 Factor), one poor factor (1 Factor), and two poor factors (2 Factor) $(\mathrm{P}=0.002)$.

survival (months after surgery in 16 deceased patients) were evaluated.
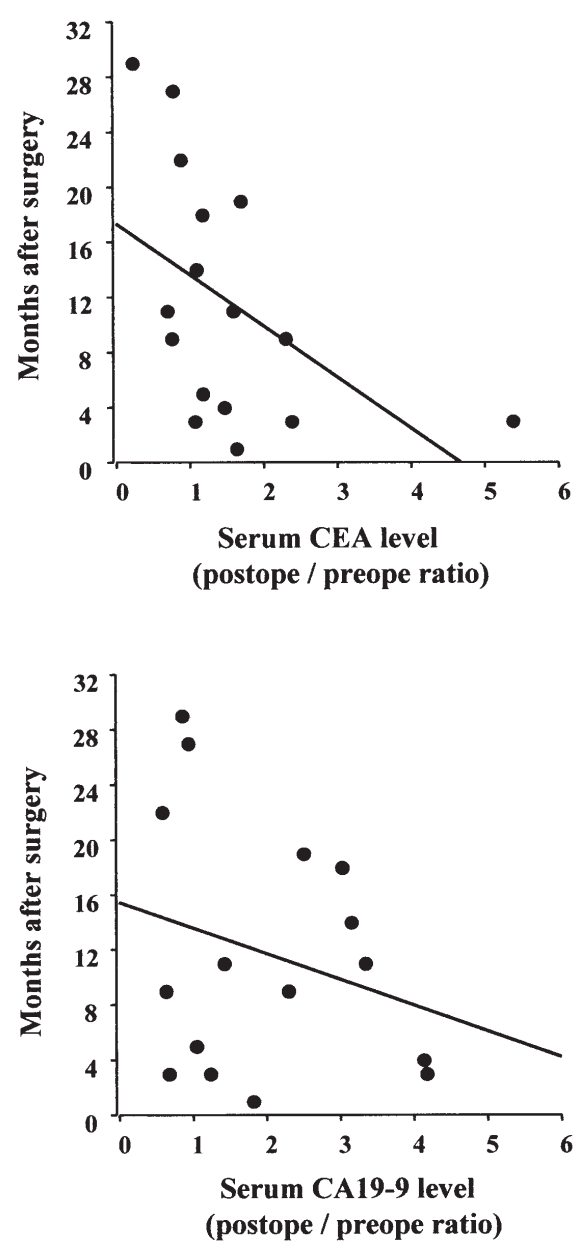

Figure 3. Correlation between the tumor markers and survival. There was a tendency to correlate between the postoperative serum CEA level/preoperative serum CEA level ratio and survival $(\mathrm{R}=-0.492, \mathrm{P}=0.053$; $y=17.388-3.733 x)$. There was a no significant correlation between the postoperative serum CA19-9 level/preoperative serum CA19-9 level and survival $(R=-0.262, P=0.327 ; y=15.457-1.866 x)$.

There was a tendency for a correlation between the postoperative serum CEA level/pre-operative serum CEA level ratio and survival $(\mathrm{R}=-0.492, \mathrm{P}=0.053 ; \mathrm{y}=17.388-3.733 \mathrm{x})$. On the other hand, there was no significant correlation between the post-operative serum CA19-9 level/preoperative serum CA19-9 level and survival $(\mathrm{R}=-0.262, \mathrm{P}=0.327$; $\mathrm{y}=15.457$ 1.866x) (Fig. 3).

\section{Discussion}

Recently, technical advances have made it easier to perform colorectal surgery with a reduced mortality rate $(1,2)$. Patients with localized and early-stage disease have an excellent survival rate after a curative resection, but the survival for patients with distant metastatic disease remains dismal (13). Liver resection has been recognized as the best treatment to offer long-term survival to patients with colorectal liver metastases $(4,7)$, but a liver resection can be performed only in a limited number of patients due to unresectable multiple and bilobar metastasis (7). In this study, we demonstrated some of the potential predictors of survival after surgery for colorectal cancer patients with synchronous unresectable MBCLM. 
Some reports have discussed the predictors of long-term survival for patients with colorectal liver metastases; AstlerColler stage A-B, negative serosa infiltration, negative peritumoral lymph node invasion as primary colorectal tumor characteristics, tumor clearance $(\geq 1 \mathrm{~cm})$ as metastatic liver tumor characteristics (14), disease-free interval ( $<12$ months), number of metastatic liver tumors $(>1)$, largest liver tumor size $(>10 \mathrm{~cm})$, positive liver resection margin, positive extrahepatic disease $(15,16)$, the presence of satellite metastases of the liver, primary tumor grade (III or IV), the time of metastasis diagnosis (synchronous), diameter of the largest liver metastasis ( $\geq 5 \mathrm{~cm}$ ) and non-anatomic approach (17).

Other reports have demonstrated that invasion or infiltration to the bile duct, portal vein, hepatic vein and perineural (18), confluent nodular type compared with simple nodular type (19), negative macroscopic fibrotic capsule formation around liver metastasis compared with a positive one $(20,21)$ and the presence of entrapped liver cells in hepatic metastases (22) were also factors of poor prognosis.

In this study, factor of the number of liver metastasis was divided into two groups; over 10 or under 10 metastasis, because cut-offs of number of liver metastasis in previous studies have been reported to be under 5 metastasis $(4,15)$, whereas, the number of liver metastasis in this study were 5 or more lesion metastases, and under 10 metastasis were considered to be accurately countable by CT and US. Factor of the maximum size of liver metastasis was divided into two groups; larger or smaller than $100 \mathrm{~mm}$, because smaller than $100 \mathrm{~mm}$ was considered to be accurately measurable by CT and US, and cut-off of the maximum size of liver metastasis in previous studies have been reported to be $100 \mathrm{~mm}(4,15)$. In this study, we did not evaluate the volume of metastatic liver disease because of its unresectability.

In our study, in univariate analysis, survival in patients with lower number of liver metastases $(\leq 10)$, without lung metastasis and peritoneal invasion, and with a $<1.0$ ratio of postoperative CEA/preoperative CEA was longer than those without them. Moreover, in multivariate analysis, the number of liver metastases $(>10)$ and a $>1.0$ ratio of postoperative CEA/ preoperative CEA were factors of poor prognosis, and patients with two poor factors had the poorest prognosis. In previous study of rescue surgery for MBCLM, patients with the more factors had also resulted in the poorer prognosis (4).

These data may contribute to developing a treatment for MBCLM, such as estimation of survival of liver resection in patients with prognostic factor improved by neoadjuvant chemotherapy.

Tumor markers were also predictive factors of survival. CEA is usually regarded as a better indicator of a poor prognosis and the recurrence of colorectal cancer (4). Some authors stated that the preoperative serum CEA level was important as a prognostic factor in patients with metastatic liver tumors $(8,16,17,23)$, others stated the postoperative level was more important than the preoperative level (24). On the other hand, Adam et al reported that a high level of preoperative serum CA19-9 (>100 IU/1) was a risk factor of survival and of disease-free survival after a liver resection (4).
In this study, there was a tendency for a correlation between the ratio of postoperative serum CEA/preoperative serum CEA and survival. A high level of postoperative serum CEA compared with the preoperative serum CEA level was considered to be a predictive factor.

Many reports have demonstrated that recent chemotherapy including leucovorin (LV) and 5-fluorouracil (5FU) combined with irinotecan (CPT-11) or oxaliplatin have survival benefits for patients with advanced colorectal cancer including unresectable liver metastasis (25-28), but the survival impact of hepatic arterial infusion chemotherapy is still controversial (29-32). The strategy for patients with unresectable liver metastases after neoadjuvant chemotherapy has been a major breakthrough (4). Secondary hepatic resection and two-stage hepatectomy after neoadjuvant chemotherapy have been reported $(33,34)$. Furthermore, in order to induce compensatory hypertrophy of the small future remnant liver and improve the mortality of this new strategy, preoperative portal vein embolization has been proposed $(7,35)$. In this study, there were no cases with secondary hepatic resection or two-stage hepatectomy after chemotherapy. The accurate indication and effects of these strategies should be demonstrated in a future trial. In addition, the lack of significant correlation may be due to the small number of patients enrolled.

Thus, we clarified some of the potential predictors of survival after surgery for colorectal cancer patients with synchronous unresectable MBCLM and the usefulness of serum CEA level.

\section{References}

1. Le Voyer TE, Sigurdson ER, Hanlon AL, et al: Colon cancer survival is associated with increasing number of lymph nodes analyzed: a secondary survey of intergroup trial INT-0089. J Clin Oncol 21: 2912-2919, 2003.

2. Hotta T, Takifuji K, Arii K, Yokoyama S, Matsuda K, Higashiguchi T, Tominaga T, Oku Y and Yamaue H: Toxicity during $1-\mathrm{LV} / 5 \mathrm{FU}$ adjuvant chemotherapy as a modified RPMI regimen for patients with colorectal cancer. Oncol Rep 14: 433-439, 2005.

3. Stangl R, Altendolf-Hofmann A, Charnley RM, et al: Factors influencing the natural history of colorectal liver metastases. Lancet 343: 1405-1410, 1994.

4. Adam R, Delvart V, Pascal G, et al: Rescue survey for unresectable colorectal liver metastases downstaged by chemotherapy. A model to predict long-term survival. Ann Surg 240: 644-658, 2004.

5. Scheele J, Stang R, Altendorf-Hofmann A and Paul M: Resection of colorectal liver metastases. World J Surg 19: 59-71, 1995.

6. Rodgers MS and NcCall JL: Surgery for colorectal liver metastases with hepatic lymph node involvement: a systematic review. Br J Surg 87: 1142-1155, 2000.

7. Jaeck D, Oussoultzoglou E, Rosso E, Greget M, Weber JC and Bachellier P: A two-stage hepatectomy procedure combined with portal vein embolization to achieve curative resection for initially unresectable multiple and bilober colorectal liver metastases. Ann Surg 240: 1037-1051, 2004.

8. Cady B, Stone MD and McDermott W: Technical and biological factors in disease-free survival after hepatic resection for colorectal cancer metastases. Arch Surg 127: 561-569, 1992.

9. UICC: TNM classification of malignant tumors. 4th edition. Berlin Heidelberg, Springer-Verlag, New York, 1997.

10. Japanese Society for Cancer of the Colon and Rectum: Japanese classification of colorectal carcinoma. 1st English edition. Kanehara Co., Ltd., Tokyo, 1997.

11. Petrelli N, Douglass HO, Herrera L, et al: The modulation of fluorouracil with leucovorin in metastatic colorectal carcinoma: a prospective randomized phase III trial. J Clin Oncol 7: 1419-1426, 1989. 
12. Petrelli N, Herrera L and Rustum Y, et al: A prospective randomized trial of 5-fluorouracil versus 5-fluorouracil and high-dose leucovorin versus 5-fluorouracil and methotrexate in previously untreated patients with advanced colorectal carcinoma. J Clin Oncol 5: 1559-1565, 1987.

13. Dhar DK, Yoshimura H, Kinukawa N, et al: Metastatic lymph node size and colorectal cancer prognosis. J Am Coll Surg 200: 20-28, 2005.

14. Jaeck D, Bachellier $\mathrm{P}$, Guiguet $\mathrm{M}$, et al: Long-term survival following resection of colorectal hepatic metastases. Br J Surg 84: 977-980, 1997.

15. Fong Y, Cohen AM, Fortner JG, et al: Liver resection for colorectal metastases. J Clin Oncol 15: 938-946, 1997.

16. Nordlinger B, Guiguet M, Vaillant JC, et al: Surgical resection of colorectal carcinoma metastases to the liver. A prognostic scoring system to improve case selection, based on 1568 patients. Cancer 77: 1254-1262, 1996.

17. Minagawa M, Makuuchi M, Torzilli G, et al: Extension of the frontiers of surgical indications in the treatment of liver metastases from colorectal cancer. Long-term results. Ann Surg 231: 487-499, 2000.

18. Okano K, Yamamoto J, Moriya Y, et al: Macroscopic intrabiliary growth of liver metastases from colorectal cancer. Surgery 126: 829-834, 1999.

19. Yasui K, Hirai T, Kato T, et al: A new macroscopic classification predicts prognosis for patient with liver metastases from colorectal cancer. Ann Surg 226: 582-586, 1997.

20. Ambiru S, Miyazaki M, Isono T, et al: Hepatic resection for colorectal metastases. Analysis of prognostic factors. Dis Colon Rectum 42: 632-639, 1999.

21. Lunevicius R, Nakanishi H, Ito S, et al: Clinicopathological significance of fibrotic capsule formation around liver metastasis from colorectal cancer. J Cancer Res Clin Oncol 127: 193-199, 2001.

22. Koike M, Yasui K, Torii A, et al: Prognostic significance of entrapped liver cells in hepatic metastases from colorectal cancer. Ann Surg 232: 653-657, 2000.

23. Fong Y, Fortner J, Ba RLS, Brennan MF and Blumgart LH: Clinical score for predicting recurrence after hepatic resection for metastatic colorectal cancer. Analysis of 1001 consecutive cases. Ann Surg 230: 309-321, 1999.
24. Hohenberger P, Schlag PM, Gerneth T and Herfarth C: Pre- and postoperative carcinoembryonic antigen determinations in hepatic resection for colorectal metastases. Ann Surg 219: 135-143, 1994.

25. Saltz LB, Cox JV, Blanke C, et al: Irinotecan plus fluorouracil and leucovorin for metastatic colorectal cancer. N Engl J Med 343: 905-914, 2000.

26. Douillard JY, Cunningham D, Roth $\mathrm{AD}$, et al: Irinotecan combined with fluorouracil compared with fluorouracil alone as first-line treatment for metastatic colorectal cancer: a multicentre randomised trial. Lancet 355: 1041-1047, 2000.

27. De Gramont A, Vignoud J, Tournigand C, et al: Oxaliplatin with high-dose leucovorin and 5-fluorouracil 48-h continuous infusion in pretreated metastatic colorectal cancer. Eur J Cancer 33: 214-219, 1997.

28. Poston GJ: The use of irinotecan and oxaliplatin in the treatment of advanced colorectal cancer. Eur J Surg Oncol 31: 325-330, 2005.

29. Martin JK Jr, O'Connell MJ, Wieand HS, et al: Intra-arterial floxuridine vs. systemic fluorouracil for hepatic metastases from colorectal cancer: a randomized trial. Arch Surg 125: 1022-1027, 1990.

30. Kemeny N, Gonen M, Sullivan D, et al: Phase I study of hepatic arterial infusion of floxuridine and dexamethasone with systemic irinotecan for unresectable hepatic metastases from colorectal cancer. J Clin Oncol 19: 2687-2695, 2001

31. Kemeny N, Huang Y, Cohen AM, et al: Hepatic arterial infusion of chemotherapy after resection of hepatic metastases from colorectal cancer. N Engl J Med 341: 2039-2048, 1999.

32. Lorenz M, Muller HH, Schramm H, et al: Randomized trial of surgery versus surgery followed by adjuvant hepatic arterial infusion with 5-fluorouracil and folinic acid for liver metastases of colorectal cancer. Ann Surg 228: 756-762, 1998

33. Bismuth H, Adam R, Lévi F, et al: Resection of nonresectable liver metastases from colorectal cancer after neoadjuvant chemotherapy. Ann Surg 224: 509-522, 1996.

34. Adam R, Laurent A, Azoulay D, Castaing D and Bismuth H: Two-stage hepatectomy: a planned strategy to treat irresectable liver tumors. Ann Surg 232: 777-785, 2000.

35. Shimada H, Tanaka K, Masui H, et al: Results of surgical treatment for multiple ( $\geq 5$ nodules) bi-lobar hepatic metastases from colorectal cancer. Langenbecks Arch Surg 389: 114-121, 2004. 